\title{
Geometric potentials for subrecoil dynamics
}

\author{
P. M. Visser and G. Nienhuis \\ Huygens Laboratorium, Rijksuniversiteit Leiden, Postbus 9504, 2300 RA Leiden, The Netherlands
}

(Received 9 January 1998)

\begin{abstract}
Quantum motion of atoms in light fields is described on the basis of adiabatic internal states. Forces arise due to the spatial variation of these states, which is determined by the electric field polarization. In a dark state, these are the only forces present. They are described by a geometric vector and a scalar potential. We give analytical expressions for the geometric potentials in the dark states of a driven $j \rightarrow j-1$ transition and the dark state in the $1 \rightarrow 1$ system, for arbitrary electromagnetic fields. For systems with velocity selective trapping states, the scalar geometric potential is inversely proportional to the field intensity squared. When the field has nodes the potential diverges. In one dimension, this constitutes an exact realization of the Kronig-Penney model. [S1050-2947(98)01806-X]
\end{abstract}

PACS number(s): 32.80.Pj, 03.65.Ge, 42.50.Vk

\section{INTRODUCTION}

In laser cooling situations, the random photon recoil of an atom at spontaneous emission increases the temperature. In order to cool below the recoil limit, spontaneous emissions must be avoided. This can be achieved when stationary states within the ground level exist. These dark states form the key ingredients for velocity-selective coherent population trapping (VSCPT) [1]. This method allows cooling below recoil temperatures and spatial coherences larger than an optical wavelength. Another advantage of trapping atoms in dark states is that the long-range dipole-dipole interaction vanishes. This becomes important at high densities, where quantum-statistical effects can be studied.

In general, atoms moving in a light-shift potential are also subject to gauge forces, which arise from the adiabatic motion in light fields with polarization gradients [2]. The corresponding potentials are of the order of the recoil energy and in most cases they can safely be ignored compared with the light shifts. In dark states, however, the light shift vanishes and the gauge potentials become important. They depend only on the field pattern, not on the overall intensity or the atom-light detuning. In this sense, the potentials have a geometric nature.

The force arising from these potentials determines the atomic motion below the recoil limit. In this paper we study the general structure of the geometric potentials. We demonstrate that they can be used to confine atoms. More generally, the geometric potentials are the main ingredients for subrecoil dynamics, which determines the final stage of VSCPT.

\section{QUANTUM MOTION AND SEMICLASSICAL FORCE}

\section{A. Transformation to adiabatic states}

We consider an atom in a classical monochromatic radiation field, which drives the transition between a degenerate ground and excited level. In the absence of dissipation, the system is described by a fully quantum-mechanical Hamiltonian consisting of the center-of-mass kinetic energy and an effective interaction term

$$
\boldsymbol{H}=\frac{1}{2 m} \overrightarrow{\mathbf{p}}^{2}+\boldsymbol{V}
$$

For a monochromatic field $\vec{E}(\vec{x})$ driving a transition between a degenerate ground and excited level, the interaction Hamiltonian $\boldsymbol{V}$ consists of the internal electronic energy levels and the dipole interaction energy. In the rotating-wave approximation, the time dependence is transformed away. Then the interaction can be diagonalized at each position as

$$
V\left|\alpha_{i}, \vec{x}\right\rangle=\left|\alpha_{i}, \vec{x}\right\rangle V_{i}(\vec{x})
$$

where the internal states $\left|\alpha_{i}\right\rangle=\left|\alpha_{i}(\vec{x})\right\rangle$ are positiondependent linear combinations of the atomic energy levels. As a function of position, the eigenvalues $V_{i}(\vec{x})$ form effective energy potentials for a moving atom, which are known as light-shift potentials. This has become the foundation of the interpretation of sub-Doppler laser cooling in terms of the Sisyphus mechanism. Often it is assumed that the atom has a well-localized wave packet and the motion is described semiclassically. We are interested in the quantum motion of cold atoms, where the semiclassical description is inadequate.

The spatial variation of the adiabatic states can be transformed away by the local transformation operator $\boldsymbol{T}$, defined by

$$
\boldsymbol{T}\left|\alpha_{i}, \vec{x}\right\rangle=|i\rangle|\vec{x}\rangle
$$

Here the set $|i\rangle$ is a fixed $\vec{x}$-independent basis of the internal state space. A natural choice would be to take $|i\rangle$ as the state corresponding to $\left|\alpha_{i}\right\rangle$ in the limit of zero field at a fixed position. This operator relates the new transformed state to the original state according to

$$
\left|\psi^{\prime}\right\rangle=\boldsymbol{T}|\psi\rangle
$$

The original state $|\psi\rangle$ and the transformed state $\left|\psi^{\prime}\right\rangle$ are explicitly given in terms of the wave functions in the adiabatic component by 


$$
\begin{aligned}
|\psi\rangle & =\sum_{i} \int d \vec{x}\left|\alpha_{i}, \vec{x}\right\rangle \psi_{i}(\vec{x}), \\
\left|\psi^{\prime}\right\rangle & =\sum_{i}|i\rangle \int d \vec{x}|\vec{x}\rangle \psi_{i}(\vec{x}) .
\end{aligned}
$$

The evolution of the transformed state is governed by the transformed Hamiltonian

$$
\boldsymbol{H}^{\prime}=\boldsymbol{T} \boldsymbol{H} \boldsymbol{T}^{\dagger}=\frac{1}{2 m}(\overrightarrow{\mathbf{p}}+\overrightarrow{\mathbf{A}})^{2}+\boldsymbol{V}^{\prime}
$$

Here the scalar and vector potentials are position-dependent operators on the internal states, as described by

$$
\boldsymbol{V}^{\prime}=\sum_{i} V_{i}(\vec{x})|i\rangle\left\langle i\left|, \quad \overrightarrow{\mathbf{A}}=\sum_{i} \sum_{j} \vec{A}_{i j}(\vec{x})\right| i\right\rangle\langle j|,
$$

with

$$
\vec{A}_{i j}(\vec{x})=-i\left\langle\alpha_{i}|\vec{\nabla}| \alpha_{j}\right\rangle=i\left(\vec{\nabla}\left\langle\alpha_{i}\right|\right)\left|\alpha_{j}\right\rangle .
$$

The scalar potential $\boldsymbol{V}^{\prime}$ is diagonal on the basis set $|i\rangle$, but the vector potential $\vec{A}$ has both diagonal and off-diagonal contributions. Notice that all these matrix elements serve as operators for the translational degrees of freedom. The offdiagonal terms of $\vec{A}$ describe the nonadiabatic coupling between different adiabatic states. For sufficiently low atomic velocities, this coupling is small compared to the energy difference between the light-shift potentials $V_{i}(\vec{x})$ and can often be neglected. The diagonal contributions, however, have to be compared with the variations of a single potential $V_{i}(\vec{x})$. The internal states are determined up to a position-dependent phase factor. This phase factor fixes the gauge, which only affects the diagonal elements of the vector potential $\vec{A}$. For this reason the vector potential is also called a gauge potential [2].

\section{B. Lorentz force and light-shift potentials}

Generally, the force on an atom is described by the force operator. In the Heisenberg picture, where the operators rather than the state vector evolve in time, the force operator is

$$
\overrightarrow{\mathbf{F}}=m \frac{d^{2} \overrightarrow{\mathbf{x}}}{d t^{2}}=-\vec{\nabla} \boldsymbol{V}
$$

The effect of the force operator is determined by its action on the adiabatic internal states, which is given by

$$
\overrightarrow{\mathbf{F}}\left|\alpha_{i}, \vec{x}\right\rangle=-\left|\alpha_{i}, \vec{x}\right\rangle \vec{\nabla} V_{i}+i \sum_{j}\left|\alpha_{j}, \vec{x}\right\rangle\left(V_{j}-V_{i}\right) \vec{A}_{j i}
$$

The diagonal elements are the gradients of the light-shift potentials. Since the off-diagonal elements are proportional to the vector potential $\vec{A}$, they are only present when the internal states depend on position. They will contribute to the force if there exist coherences between different adiabatic components.
The transformation $\boldsymbol{T}$ to the adiabatic basis is a local transformation on the internal states. Therefore, the expectation value of the position, velocity, and acceleration is invariant under this transformation. Due to the appearance of the vector potential, the velocity and acceleration operators, however, have a different form. In the primed frame, the velocity operator follows from the Heisenberg equation

$$
\overrightarrow{\mathbf{p}}^{\prime}=m \frac{d \overrightarrow{\mathbf{x}}^{\prime}}{d t}=\overrightarrow{\mathbf{p}}+\overrightarrow{\mathbf{A}}
$$

The force operator is proportional to the acceleration

$$
\overrightarrow{\mathbf{F}}^{\prime}=m \frac{d^{2} \overrightarrow{\mathbf{x}}^{\prime}}{d t^{2}}=-\vec{\nabla} \boldsymbol{V}^{\prime}-\frac{1}{2} \frac{d \overrightarrow{\mathbf{x}}^{\prime}}{d t} \times \overrightarrow{\mathbf{B}}+\frac{1}{2} \overrightarrow{\mathbf{B}} \times \frac{d \overrightarrow{\mathbf{x}}^{\prime}}{d t} .
$$

Here the vector field operator $\overrightarrow{\mathbf{B}}=\vec{\nabla} \times \overrightarrow{\mathbf{A}}$, just as in the case of a charged particle in a magnetic field. The vector operator $\overrightarrow{\mathbf{B}}$ depends on position and acts on the atomic internal states. The gradient of the potential operator $\boldsymbol{V}^{\prime}$ does not couple different internal states since

$$
-\vec{\nabla} V^{\prime}|i\rangle|\vec{x}\rangle=-|i\rangle|\vec{x}\rangle \vec{\nabla} V_{i}
$$

\section{Adiabatic approximation}

In an electric field of high intensity, atoms experience strong light shifts and the potentials $V_{i}$ are separated. When the potential curves are sufficiently different, the atoms can be confined within a single adiabatic state $\left|\alpha_{i}\right\rangle$ with potential $V_{i}$, as long as their velocity is not so fast that tunneling to other potentials becomes possible. Near a crossing, the adiabatic approximation breaks down. When a single adiabatic state $\left|\alpha_{i}\right\rangle$ is populated, the state is determined by a single wave function. Then the internal state is not a dynamical variable anymore, but a fixed quantity. The total state $|\psi\rangle$ and the transformed state $\left|\psi^{\prime}\right\rangle$ are explicitly given by this single component in the state (2.3)

$$
|\psi\rangle=\int d \vec{x}\left|\alpha_{i}, \vec{x}\right\rangle \psi(\vec{x}), \quad\left|\psi^{\prime}\right\rangle=|i\rangle \int d \vec{x}|\vec{x}\rangle \psi(\vec{x})
$$

The quantum-mechanical motion of atoms is then governed by an approximate Hamiltonian $\boldsymbol{H}_{i}$, which is basically the projection of $\boldsymbol{H}^{\prime}$ on the state $|i\rangle$. This effective Hamiltonian is defined by its action on the wave function $\psi(\vec{x})$ in terms of the full and the transformed Hamiltonians (2.1) and (2.4) by

$$
\boldsymbol{H}_{i} \psi(\vec{x})=\left\langle\alpha_{i}, \vec{x}|\boldsymbol{H}| \psi\right\rangle=\langle i|\left\langle\vec{x}\left|\boldsymbol{H}^{\prime}\right| \psi^{\prime}\right\rangle
$$

Thus the effective Hamiltonian is written explicitly in the position representation as

$$
\boldsymbol{H}_{i}=\frac{1}{2 m}\left[-i \vec{\nabla}+\vec{A}_{i}(\vec{x})\right]^{2}+U_{i}(\vec{x})+V_{i}(\vec{x})
$$

where the momentum operator is represented as $-i \vec{\nabla}$. The vector and scalar potentials are 


$$
\vec{A}_{i}=\vec{A}_{i i}, \quad U_{i}=\frac{1}{2 m} \sum_{j \neq i}\left|\vec{A}_{i j}\right|^{2} .
$$

The vector potential $\vec{A}_{i}$ is the diagonal matrix element of the vector operator. The scalar potential $U_{i}$ arises from the offdiagonal terms in $\overrightarrow{\mathbf{A}}$ that contribute to the diagonal part of $\boldsymbol{H}^{\prime}$. They describe the kinetic energy that can be associated with the variation of the internal state. At positions where the couplings from $|i\rangle$ to all the other internal states is zero the potential $U_{i}$ vanishes. When the light-shift potentials $V_{i}$ are degenerate, the effective Hamiltonian $\boldsymbol{H}_{i}$ acts on the corresponding subspace of states $|i\rangle$ with the same potential. Then $U_{i}$ and $\vec{A}_{i}$ should be replaced by operators on this subspace. Within this subspace, these operators are not necessarily diagonal.

Spontaneous emissions can put an atom into a superposition of different internal adiabatic states (2.3) instead of a single adiabatic state as in Eq. (2.6). Then the evolution in the adiabatic approximation is governed by an effective Hamiltonian, which is simply the sum of Hamiltonians (2.7) acting on the different components. For instance, the scalar potentials are described by the operator $\boldsymbol{U}=\Sigma_{i} U_{i}|i\rangle\langle i|$.

The magnetic field $\overrightarrow{\mathbf{B}}$ and the scalar potential $\boldsymbol{U}$ are local operators, i.e., they act on the internal states and are functions of the position operator, not of the momentum operator. This implies that it is possible to make a semiclassical description of the average force on the atom, which is valid when the wave functions are sufficiently localized. This is a bit surprising since these effects originate from the momentum operator in the Hamiltonian and thus can be considered as pure quantum forces. In the adiabatic approximation, the semiclassical force on the atom is

$$
\vec{F}=-\vec{\nabla} V-\vec{\nabla} U-\frac{d \vec{x}}{d t} \times \vec{B}
$$

Here $V, U$, and $\vec{B}$ are the local expectation values of $\boldsymbol{V}, \boldsymbol{U}$, and $\overrightarrow{\boldsymbol{B}}$ with respect to the internal-state density operator. When the rate of optical pumping is high compared to the field variation that the moving atom experiences, the internal state will follow the local steady state. When the field varies appreciably over a wavelength, this requires that the pumping rate exceeds the Doppler width. Below the recoil limit, where the semiclassical description breaks down, the steadystate assumption is well justified. Simple analytical expressions for the internal steady state can be found in $[3,4]$. In the subsequent sections, we consider delocalized wave functions.

\section{Internal dark states}

For intensities high enough to justify the adiabatic approximation, the effect of the light-shift potential $V_{i}$ is usually considerably stronger than that of the geometric potentials $\vec{A}_{i}$ and $\vec{U}_{i}$. Obviously, this is not true when $V_{i}$ is independent of $\vec{x}$, which is the case for a dark state. Dark internal states are eigenstates $\left|\alpha_{0}(\vec{x})\right\rangle$ of $\boldsymbol{V}$ that are linear combinations of substates of the ground level. If one or more dark states are present, a localized atom will eventually be
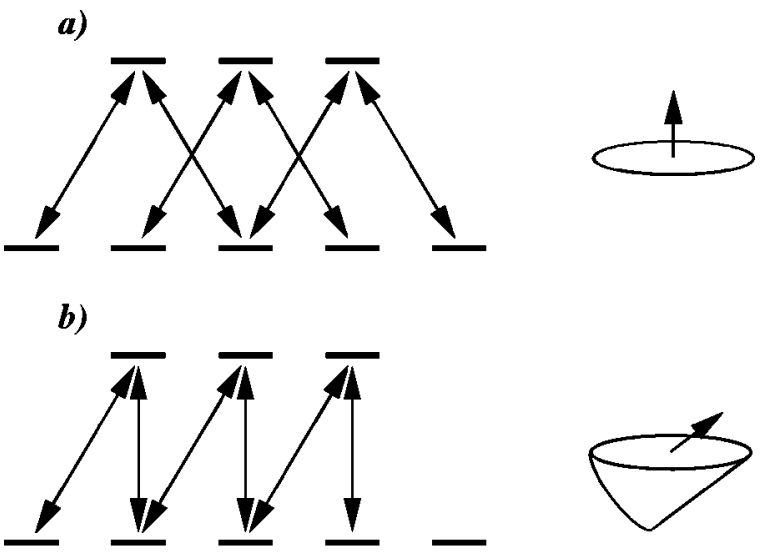

c)
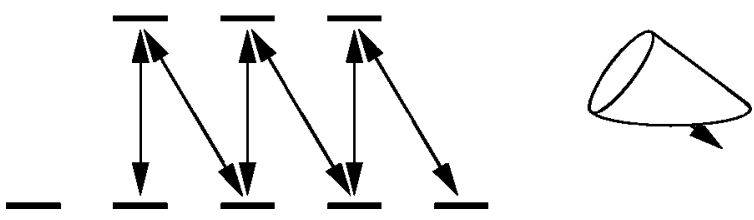

FIG. 1. Two dark states in the $j \rightarrow j-1$ transition. (a) Level scheme when the orientation of the quantization axis is orthogonal to the plane of the polarization ellipse. (b) and (c) Situations where the axis is determined by the cylinders on the polarization ellipse.

pumped into such a dark state. Insensitive to the light field and with no possible escape by spontaneous decay, an atom will stay in the dark state until it moves nonadiabatically to other states. Very slowly moving atoms can be confined in the dark state for times long compared to the optical pumping time. This means that the adiabatic approximation is valid. In a dark state, the light-shift potential vanishes. The translational dynamics of the atom in a dark state is entirely determined by the potentials $\vec{A}_{0}$ and $U_{0}$. We shall denote the dark state with the index $i=0$.

Since no light shifts occur, the adiabatic potential of a dark state is flat: $V_{0}(\vec{x})=0$. Therefore, periodic optical lattices, trapping, and Sisyphus cooling in a dark state are thought not to be possible. So-called gray lattices have been proposed [7] to create a periodic adiabatic potential by means of adding a small magnetic field. Unfortunately, then small excited state amplitudes are added and dark states disappear. However, it follows from the previous discussion that position-dependent forces can arise in dark adiabatic states, due to the position dependence of the dark state $\left|\alpha_{0}(\vec{x})\right\rangle$. Since the dark state is determined only by the field polarization and not by the field intensity or atom-light detuning, we call the vector and the scalar potentials for dark states geometric potentials.

The simplest model of a dark internal state is a $\Lambda$ configuration. For particular values of the two ground-state amplitudes the coupling to the excited state is canceled by destructive interference [5]. In terms of an arbitrary elliptical polarization $\hat{\mathcal{E}}$, the dark states in a $j \rightarrow j^{\prime}$ transition can be found by choosing the atomic quantization axis orthogonal to the polarization plane. This implies that arrows coupling the ground state $|j, m, g\rangle$ to the state $\left|j^{\prime}, m, e\right\rangle$ with the same $m$ by linearly polarized light disappear [see Fig. 1(a)]. The remaining multiple $\Lambda$ structure contains dark states provided 
a)

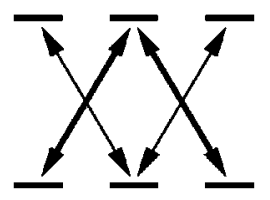

b)

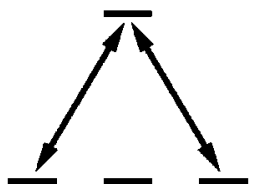

c)

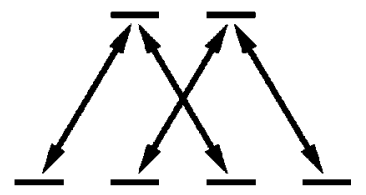

d)

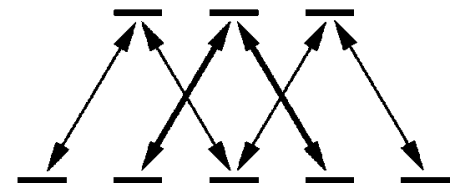

FIG. 2. Black states in one-dimensional systems. Multiple $\Lambda$ structures contain a dark internal state. In a single $\Lambda$ (plotted with bold arrows) a full black state is found. (a) The $1 \rightarrow 1$ transition contains one dark internal state and one black state. (b) The $1 \rightarrow 0$ transition contains two dark states: The $\Lambda$ has one black state, the isolated state is completely black. (c) In $3 / 2 \rightarrow 1 / 2$ two $\Lambda$ 's are present; hence there are two dark states with a black state each. (d) In $2 \rightarrow 1$ there are two dark states, one with a black state.

that the ends of the wiggle are in the ground level.

When an atomic transition between levels with angular momentum $j$ and $j^{\prime}$ is driven by light with an arbitrary polarization, two dark states exist when $j^{\prime}=j-1$. When $j^{\prime}$ $=j$, there is a single dark state for integer $j$ values. For half-integer values of $j$, a single dark state only exists when the polarization is circular [6].

\section{E. Black states}

A full quantum-mechanical dark state is denoted as in Eq. (2.6) with $i=0$ for an arbitrary wave function $\psi(\vec{x})$. Although such dark states are eigenstates of the interaction Hamiltonian, they are stationary states only when they are also eigenstates of the kinetic energy and the full Hamiltonian (2.1). Time evolution will dephase the components of the state with different kinetic energy or, equivalently, the wave packet will be deformed. As a result, bright states will become occupied. Hence atomic states in the dark internal state can still decay. In the transformed basis, this process is described by the operators $\vec{A}_{i 0}$, which couple the dark state to bright states. Only in exceptional cases can a dark state be stationary. These eigenstates of the complete Hamiltonian in the ground state will be called black states.

An exact black state $\left|\psi_{0}\right\rangle$ is found for an arbitrary field in the transition between two levels with $j=1$ and two black states are present in the $1 \rightarrow 0$ transition. In the onedimensional case, a black state occurs in the transition 2 $\rightarrow 1$, since it contains a single $\Lambda$, and two black states occur in the transition $3 / 2 \rightarrow 1 / 2$, since it contains two $\Lambda$ 's (see Fig. 2 ). These are the famous velocity-selective trapping states $[1,8,9]$. The recoil kick of a spontaneous emission can put the atom in the black state, where it is trapped. Hence the population in $\left|\psi_{0}\right\rangle$ can only increase with time. The widths of the momentum distribution can become smaller than a single recoil, resulting in very low temperatures.

\section{A SINGLE DARK STATE}

In order to demonstrate the importance of the geometric potentials $\vec{A}_{0}$ and $U_{0}$, we give explicit expressions in a num-

ber of specific cases of physical relevance in this and the following sections. We notice that on a $j=1$ level, there exists a basis that transforms under rotations like a Cartesian basis $|\hat{x}\rangle,|\hat{y}\rangle,|\hat{z}\rangle$. This implies that each internal state can be represented as a vector, which we denote as

$$
|\vec{a}\rangle=a_{x}|\hat{x}\rangle+a_{y}|\hat{y}\rangle+a_{z}|\hat{z}\rangle
$$

We consider a $1 \rightarrow 1$ transition. In this case an exact black state is known to exist [8]. The two polarization vectors $\vec{a}$ and $\vec{b}$ of the ground and excited state are coupled by the field $\vec{E}$. The interaction operator acting on an arbitrary ground state $|\vec{a}, g\rangle$ is then determined by the vector product of the fields and atomic polarization vectors

$$
\langle\vec{b}, e, \vec{x}|\boldsymbol{V}| \vec{a}, g, \vec{x}\rangle=\kappa \vec{b}^{*} \cdot(\vec{E} \times \vec{a})
$$

since this is the only linear operation that forms a vector out of two vectors. Here $\kappa$ is a coupling constant. The interaction with the light field vanishes with $\vec{a}=\hat{\mathcal{E}}$, where $\hat{\mathcal{E}}$ is the normalized polarization vector and $E$ is the real amplitude of the electric field $\vec{E}=E \hat{\mathcal{E}}$. This shows that dark states are characterized by the internal state $\left|\alpha_{0}\right\rangle=|\hat{\mathcal{E}}, g\rangle$.

\section{A. Wave function of the black state}

An exact black state

$$
\left|\psi_{0}\right\rangle=\int d \vec{x}|\hat{\mathcal{E}}, g, \vec{x}\rangle \psi_{0}(\vec{x})
$$

is obtained when $\psi_{0}(\vec{x})$ is chosen such that $\left|\psi_{0}\right\rangle$ is an eigenstate of the kinetic energy $\overrightarrow{\mathbf{p}}^{2} / 2 \mathrm{~m}$. An obvious choice that realizes this is $\psi_{0}(\vec{x})=E(\vec{x})$, the electric field amplitude [8]. This can be shown when one notices that the electric field is a solution of the Helmholtz equation

$$
-\vec{\nabla}^{2} \vec{E}=k^{2} \vec{E}
$$

Then it follows that

$$
\boldsymbol{H}\left|\psi_{0}\right\rangle=\frac{1}{2 m} \overrightarrow{\mathbf{p}}^{2}\left|\psi_{0}\right\rangle=E_{0}\left|\psi_{0}\right\rangle,
$$

with energy eigenvalue $E_{0}=k^{2} / 2 m$. It follows that the wave function $\psi_{0}(\vec{x})$ is an exact eigenfunction of the effective Hamiltonian (2.7) for the dark state

$$
\boldsymbol{H}_{0} \psi_{0}(\vec{x})=\left\langle\alpha_{0}, \vec{x}|\boldsymbol{H}| \psi_{0}\right\rangle=E_{0} \psi_{0}(\vec{x})
$$

where we used the definition (2.6). The wave function $\psi_{0}$ $=E$ is equal to the electric field amplitude. If the electric field has no nodes, $\psi_{0}$ has no zeros and then this wave function must be the lowest-energy eigenstate of $\boldsymbol{H}_{0}$. Unlike the total state $\left|\psi_{0}\right\rangle$, the corresponding wave function $\psi_{0}=E$ does not have a well-defined kinetic energy. This explains the presence of the geometric potential for the dark state in the driven $1 \rightarrow 1$ transition. 


\section{B. Geometric potential in terms of the electric field}

The vector and scalar potentials (2.8) in the adiabatic approximation as defined by Eq. (2.5) for the $1 \rightarrow 1$ transition can be expressed in the field polarization vectors. The vector potential is

$$
\vec{A}_{0}=-i \hat{\mathcal{E}}^{*} \vec{\nabla} \hat{\mathcal{E}}=i\left(\vec{\nabla} \hat{\mathcal{E}}^{*}\right) \hat{\mathcal{E}}=\frac{1}{E^{2}} \operatorname{Im} \vec{E}^{*} \vec{\nabla} \vec{E}
$$

and the scalar potential is

$$
U_{0}=\frac{1}{2 m}\left(|\vec{\nabla} \hat{\mathcal{E}}|^{2}-\vec{A}^{2}\right)=\frac{1}{2 m}|\hat{\mathcal{E}} \times \vec{\nabla} \hat{\mathcal{E}}|^{2} .
$$

The notation needs some comment. Note that it follows from the definition (2.5) that the gradient operators always combine with gradient operators in inner or outer products and field vectors $\vec{E}$ or $\hat{\mathcal{E}}$ combine with field vectors. From the second expression it can be seen that the component orthogonal to the field is picked out.

A monochromatic radiation field consists of a finite number of plane traveling waves with wave vectors $k_{i}$ and polarizations $\vec{E}_{i}$

$$
\vec{E}(\vec{x})=\sum_{i} \vec{E}_{i} e^{i \vec{k}_{i} \cdot \vec{x}}
$$

The scalar potential can now be expressed in terms of the total electric field vector $\vec{E}$ or in terms of the amplitudes $\vec{E}_{i}$ by

$$
U_{0}=\frac{1}{2 m} \frac{1}{E^{4}}|\vec{E} \times \vec{\nabla} \vec{E}|^{2}=\frac{1}{2 m} \frac{1}{E^{4}}\left|\sum_{i} \sum_{j} \vec{k}_{j} \vec{S}_{i j} e^{i\left(\vec{k}_{i}+\vec{k}_{j}\right) \cdot \vec{x}}\right|^{2}
$$

in terms of the antisymmetric matrix $\vec{S}_{i j}=\vec{E}_{i} \times \vec{E}_{j}$. The first equality of Eq. (3.3) can be verified after substituting $\vec{E}=E \hat{\mathcal{E}}$, which produces Eq. (3.2). Two different beams give a contribution to the potential only if their polarizations are different. This is understandable since the potential arises from polarization gradients. When more than two beams are present, the plane wave factors in the summation can interfere. One verifies that where $\psi_{0}$ has minima, indeed high values of the potential $U_{0}$ can be expected. In the dark state optical lattices can be created when $U_{0}$ is periodic.

The vector operator (3.1) determines an effective magnetic field. After some algebra one obtains in analogy to Eq. (3.3)

$$
\vec{B}_{0}=\vec{\nabla} \times \vec{A}_{0}=\frac{-i}{E^{4}}(\vec{E} \times \vec{\nabla} \vec{E})^{*} \times(\vec{E} \times \vec{\nabla} \vec{E})
$$

Whereas the gradient operators have an inner product in the expressions for $U_{0}$, in the expression for $\vec{B}_{0}$, the vector product between gradient operators is taken.

According to Heisenberg's uncertainty relations, an atom with a wave packet localized within a wavelength must have a momentum spread of more than one photon recoil. Hence its energy is higher than one recoil energy. The geometric nature of the scalar potential suggests that its strength is of the order of a single recoil energy. In optical lattices, the widths of the potential wells is normally smaller than a wavelength and the question arises whether strong binding in the scalar potential is possible. We show that in special configurations it can become sufficiently strong to support bound states in the potential minima.

\section{The electric field}

In order to see how the geometric potential depends on the polarization of the electric field, we expand a general field $\vec{E}=E \hat{\mathcal{E}}$ in terms of real basis vectors $\hat{u}_{1}$ and $\hat{u}_{2}$ by

$$
\hat{\mathcal{E}}=\left(\hat{u}_{1} \cos \varepsilon+i \hat{u}_{2} \sin \varepsilon\right) e^{i \varphi}
$$

The real amplitude $E$, the complex amplitude $F$, the phase $\varphi$, and the ellipticity $\varepsilon$ are determined by

$$
E^{2}=\vec{E}^{*} \cdot \vec{E}, \quad F^{2}=\vec{E} \cdot \vec{E}=E^{2} e^{2 i \varphi} \cos 2 \varepsilon .
$$

The orientation of the polarization ellipse is defined by the axes $\hat{u}_{1}$ and $\hat{u}_{2}$. The vectors $\hat{u}_{1}, \hat{u}_{2}$ are given explicitly in terms of the field vector $\vec{E}$ by

$$
\hat{u}_{1}=\frac{\vec{E}^{*} F+F^{*} \vec{E}}{2 E|F| \cos \varepsilon}, \quad \hat{u}_{2}=\frac{i \vec{E}^{*} F-i F^{*} \vec{E}}{2 E|F| \sin \varepsilon}
$$

In experimental situations, the field can be generated by superimposing counterpropagating traveling waves. In the case of two traveling waves in the $z$ direction, the field depends only on the $z$ coordinate. The general form is

$$
\vec{E}(z)=\left(\vec{E}_{+} e^{+i k z}+\vec{E}_{-} e^{-i k z}\right) / \sqrt{2} .
$$

The polarization ellipse now lies in the $x y$ plane everywhere and $\hat{u}_{1}=\hat{z}$. The polarization vector is entirely determined by the ellipticity $\varepsilon$ and the phase as defined in Eq. (3.5) and the orientation angle $\xi$. The gradient of this angle is determined by

$$
\nabla \hat{u}_{1}=\hat{u}_{2} \nabla \xi
$$

A shift in the relative phases of $\vec{E}_{ \pm}$is equivalent to a shift of the spatial coordinate. Thus, without loss of generality, we can assume that the inner product $\vec{E}_{+}^{*} \cdot \vec{E}_{-}$is real and negative, which will be advantageous in later use. The choice of phase ensures that the positions of minimal intensity are located at $k z=n \pi$. The field intensity pattern has the universal form

$$
E^{2}(z)=(1-\cos 2 \alpha \cos 2 k z) I
$$

in terms of the average intensity $I=\left(E_{+}^{2}+E_{-}^{2}\right) / 2$. The parameters $\alpha$ and $\beta$ are defined by the overlap and the cross product of the two polarizations as

$$
I \cos 2 \alpha=\left|\vec{E}_{+}^{*} \cdot \vec{E}_{-}\right|, \quad I \sin 2 \beta=\left|\vec{E}_{+} \times \vec{E}_{-}\right| .
$$

Hence $\cos 2 \alpha=0$ when the two polarizations are normal to one another and $\cos 2 \alpha=1$ when $\vec{E}_{+}=-\vec{E}_{-}$. The two parameters are related according to 


$$
\cos ^{2} 2 \alpha+\sin ^{2} 2 \beta=E_{+}^{2} E_{-}^{2} / I^{2} .
$$

\section{The atomic system}

When the field consists of two counterpropagating traveling waves, only two ground states and one excited state participate in the dynamics. The light-shift potentials (2.2) are

$$
\begin{gathered}
V_{0}=0, \quad V_{1}=-\frac{1}{2} \delta+\frac{1}{2} \sqrt{\delta^{2}+\kappa^{2} E^{2}}, \\
V_{2}=-\frac{1}{2} \delta-\frac{1}{2} \sqrt{\delta^{2}+\kappa^{2} E^{2}} .
\end{gathered}
$$

For large detuning, the excited-state population can be neglected and one has two ground states: the dark state $\left|\alpha_{0}\right\rangle$ and the bright state $\left|\alpha_{1}\right\rangle$. The scalar potentials of the twodimensional space with basis states $|0\rangle$ and $|1\rangle$ are equal. With Eqs. (3.2) and (3.8) they are expressed in the positiondependent angles $\varepsilon$ and $\xi$ that determine the field polarization (3.4) by

$$
U_{0}=U_{1}=U=\frac{1}{2 m}\left[(\nabla \xi)^{2} \cos ^{2} 2 \varepsilon+(\nabla \varepsilon)^{2}\right] .
$$

By substitution of Eq. (3.7) in Eq. (3.3) and using Eq. (3.9), we obtain an explicit expression in terms of the constant field parameters $\alpha, \beta$,

$$
U(z)=\frac{k^{2}}{2 m}\left(\frac{\sin 2 \beta}{1-\cos 2 \alpha \cos 2 k z}\right)^{2} .
$$

With the proper choice of the phase of the bright state, the diagonal matrix elements of the vector potential $A_{00}$ and $A_{11}$ are equal. They can be evaluated from the last identity in Eq. (3.1), with the field (3.7). With Eq. (2.8) it follows that the off-diagonal elements $A_{01}$ and $A_{10}$ are determined by the scalar potential up to a constant phase factor. Therefore, these matrix elements can be chosen positive. With Eq. (3.12) the result is

$$
A_{00}=A_{11}=A=k \frac{E_{+}^{2}-E_{-}^{2}}{E^{2}}, \quad A_{01}=A_{10}=k \frac{I \sin 2 \beta}{E^{2}} .
$$

The periodic potentials $V_{1}, V_{2}, U$, and $A, A_{01}$ have the lattice constant $a=\pi / k$. The vector potential $A$ is only present when the running waves have different strengths.

\section{E. Gauge transformations}

When we do not require that the amplitude $E$ is real, the separation between $E$ and $\hat{\mathcal{E}}$ is defined apart from a positiondependent phase factor. This implies that the dark internal state and the corresponding wave function also obtain the same phase factor. The transformations for the field and the wave function are

$$
\vec{E}=E \hat{\mathcal{E}}=E^{\prime} \hat{\mathcal{E}}^{\prime}=E e^{i \varphi} \hat{\mathcal{E}}^{\prime}, \quad \psi^{\prime}=\psi e^{i \varphi}
$$

The vector and scalar potentials in the new (primed) representation are related to the original potentials by

$$
\vec{A}^{\prime}=\vec{A}-\vec{\nabla} \varphi, \quad U^{\prime}=U
$$

The potentials $\vec{A}, U$ and $\vec{A}^{\prime}, U^{\prime}$ respectively correspond to the polarizations $\hat{\mathcal{E}}$ and $\hat{\mathcal{E}}^{\prime}$.

In a one-dimensional system the vector potential can always be transformed away by choosing the proper gauge. The gauge $A^{\prime}=0$ is a natural choice since then the Hamiltonian is real. It only contains the scalar potential $U$. Hence with Eq. (3.13), the equation for the phase is

$$
\nabla \varphi=A=p \frac{\sin 2 \alpha}{1-\cos 2 \alpha \cos 2 k z} \quad \text { where } \quad p=k \frac{E_{+}^{2}-E_{-}^{2}}{I \sin 2 \alpha} .
$$

The solution can be expressed as

$$
\tan \frac{k \varphi(z)}{p}=\frac{\tan k z}{\tan \alpha} .
$$

In the primed gauge, the wave function of the black state is explicitly written in terms of the vector potential $A$ by

$$
\psi_{0}^{\prime}(z)=E(z) e^{i \varphi(z)}=\sqrt{\frac{I p \sin 2 \alpha}{A(z)}} \exp \left(i \int A(z) d z\right) .
$$

It follows from the solution (3.14) that the phase shift over half an optical wavelength is $\varphi(a)-\varphi(0)=p a$. Hence the state (3.15) satisfies Bloch's theorem

$$
\psi^{\prime}(z+a)=\psi^{\prime}(z) e^{i p a}
$$

for eigenstates of the real Hamiltonian with a periodic potential $U$, with the quasimomentum $p$. The wave function lies in the lowest Bloch band since it does not have zeros. We stress that the wave function (3.15) of the black state is an exact solution of the effective Hamiltonian $\boldsymbol{H}_{0}$ with potential (3.12), as can be verified explicitly. This is remarkable since the geometric potential $U$ is only present in the adiabatic approximation.

The phase factor in the Bloch state (3.15) can be considered as a Berry phase corresponding to the vector potential $A$. Thus, in the periodic geometric potential $U$, the Berry phase describing the adiabatic following of the internal state is precisely the quasimomentum times the period. When the potential is adiabatically translated over a period $a$, the Bloch state (3.15) also obtains a Berry phase factor. That Berry phase is determined by the gradient of the translational state, which turns out to be the expectation value $\langle\boldsymbol{p}\rangle$ of the momentum operator. The quasimomentum $p$ and the real momentum (or group velocity) $\langle\boldsymbol{p}\rangle$ of a Bloch state of the general form (3.15) are given by the simple relations

$$
p a=\int_{0}^{a} A d z, \quad \frac{a}{\langle\boldsymbol{p}\rangle}=\int_{0}^{a} \frac{d z}{A} .
$$

In the Bloch state (3.15) where $A$ is determined by Eq. (3.13), the true momentum is

$$
\langle\boldsymbol{p}\rangle=p \sin 2 \alpha=k \frac{E_{+}^{2}-E_{-}^{2}}{I},
$$

which is equal to the expectation value of the momentum operator in the original black state $\left|\psi_{0}\right\rangle$ and to the average momentum of a photon in the field mode $E$. 


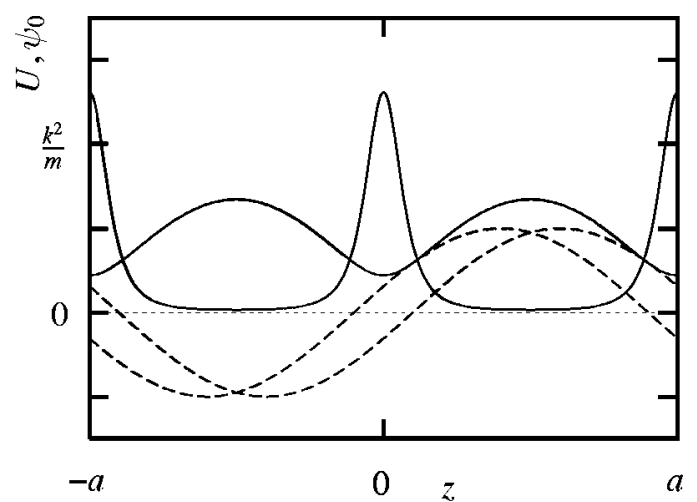

FIG. 3. Geometric potential $U$ and the ground-state wave function $\psi_{0}$ for the configuration (3.18) with $\alpha=\pi / 10$. The dashed curves are the two standing-wave components.

When the counterpropagating beams have equal intensities, the field and the black state have zero average momentum density. Then the angles $\alpha=\beta$ coincide. In the gauge where the amplitude $E$ is real, the vector potential $A$ vanishes. The ground state of the gauge potential $U$ is the Bloch state with $p=0$ in the lowest-energy band. This is precisely the black state. The potential $U$ and the wave function $\psi_{0}$ are plotted in Fig. 3 in the case that $p=0$.

\section{F. Examples}

As an example we take the field generated by two traveling waves of equal intensity with linear polarizations under angles $\pm \alpha$ with respect to the $\hat{y}$ axis. Then the field is elliptically polarized with a fixed orientation $\xi=0$. It is

$$
\begin{aligned}
\vec{E}(z) & =(\hat{x} \sin \alpha \cos k z+i \hat{y} \cos \alpha \sin k z) \sqrt{2 I} \\
& =[(\hat{x}+i \hat{y}) \sin (\alpha+k z)+(\hat{x}-i \hat{y}) \sin (\alpha-k z)] \sqrt{I / 2} .
\end{aligned}
$$

The ellipticity and its gradient are given by

$$
\tan \varepsilon(z)=\frac{\tan k z}{\tan \alpha}, \quad \nabla \varepsilon=k \frac{\sin 2 \alpha}{1-\cos 2 \alpha \cos 2 k z} .
$$

This field is linearly polarized when the intensity is minimal or maximal, which is at the location where $k z=n \pi$ and $n \pi+\pi / 2$. The field is purely circular when $k z=n \pi+\alpha$ and $n \pi-\alpha$.

A field that is linearly polarized everywhere is

$$
\begin{aligned}
\vec{E}(z) & =(\hat{x} \sin \alpha \cos k z+\hat{y} \cos \alpha \sin k z) \sqrt{2 I} \\
& =[(\hat{x}+\hat{y}) \sin (\alpha+k z)+(\hat{x}-\hat{y}) \sin (\alpha-k z)] \sqrt{I / 2} .
\end{aligned}
$$

It is generated by traveling waves with elliptic polarizations with ellipticities $\pm \alpha$ with the same orientation. The positiondependent polarization angle $\xi(z)$ is determined by

$$
\tan \xi(z)=\frac{\tan k z}{\tan \alpha}, \quad \nabla \xi=k \frac{\sin 2 \alpha}{1-\cos 2 \alpha \cos 2 k z} .
$$

For small ellipticities $\alpha$, the field is linearly polarized near the $\hat{y}$ direction almost everywhere. Then the polarization rotates rapidly from $\hat{x}$ to $\hat{y}-\hat{x}$ and to $\hat{x}$ at the intensity minimum and then to $\hat{x}+\hat{y}$ and back to $\hat{y}$.

\section{TWO DEGENERATE DARK STATES}

In a transition between a ground state with angular momentum $j$ and an excited state with angular momentum $j^{\prime}$ $=j-1$ two dark states exist. When the atomic quantization axis is along $\hat{u}_{3}$, orthogonal to the local field polarization (3.4), the couplings between the states in the ground and excited level with the same $m$ disappear. The level scheme consists of two multiple $\Lambda$ 's, each of which contains one dark state.

By choosing a different quantization axis, it is also possible to eliminate the couplings from $m$ to $m+1$ or from $m$ to $m-1$ in the level scheme. The electric field can be expressed as a superposition of an orthogonal pair of a linear and a circular polarization. The couplings of the third (circular) polarization disappear when the quantization axis is chosen in the direction of the linear polarization [6]. The two representations

$$
\begin{aligned}
\hat{\mathcal{E}} & =\left(\hat{v}_{1}+i \hat{u}_{2}\right) \sin \varepsilon+\hat{v}_{3} \sqrt{\cos 2 \varepsilon} \\
& =\left(\hat{w}_{1}+i \hat{u}_{2}\right) \sin \varepsilon+\hat{w}_{3} \sqrt{\cos 2 \varepsilon},
\end{aligned}
$$

correspond to the possibilities to use a left circular or a right circular component. This second coordinate frame is obtained by rotating the system about the axis $\hat{u}_{2}$ by an angle $\theta$. When $0<\varepsilon<\pi / 4$, this angle is determined by $\cos \theta=\tan \varepsilon$. Generally, for arbitrary values of $\varepsilon$,

$$
\cos \theta=\min (|\tan \varepsilon|,|\cot \varepsilon|) .
$$

Geometrically, the cylinder that encloses the polarization ellipse determines the orientation of this basis as is depicted in Figs. 1(b) and 1(c). In the basis of the quantization axis $\hat{v}_{3}$, the right circular polarization is eliminated. For the $j \rightarrow j$ -1 transition, this implies that the state $|j, m=+j, g\rangle$ is isolated. Hence it is a dark state.

We introduce states $|\vec{a}\rangle$ defined as the state $|j,+j\rangle$ in the basis where the quantization axis is in the direction of $\vec{a}$. These states are called Bloch states since they maximize the length of the Bloch vector, which is the expectation value of the angular momentum operator $\vec{J}$. (They have no relation with the stationary states of a periodic potential, which are also called Bloch states.)

The Bloch states $\left|\hat{v}_{3}\right\rangle$ and $\left|\hat{w}_{3}\right\rangle$ are dark. In terms of the vectors $\hat{u}_{2}$ and $\hat{u}_{3}$, these states are

$$
\left|\hat{v}_{3}\right\rangle=e^{-i \theta \overrightarrow{\mathbf{J}} \cdot \hat{u}_{2}}\left|\hat{u}_{3}\right\rangle, \quad\left|\hat{w}_{3}\right\rangle=e^{+i \theta \overrightarrow{\mathbf{J}} \cdot \hat{u}_{2}}\left|\hat{u}_{3}\right\rangle .
$$

These two dark states are linearly independent, but not orthogonal. The inner product of the Bloch states is $\left\langle\hat{v}_{3} \mid \hat{w}_{3}\right\rangle$ $=c^{2 j}$ with $c=\cos \theta$. Note that the inner product of the two vectors is $\hat{v}_{3} \cdot \hat{w}_{3}=\cos 2 \theta$.

The linear combinations 


$$
\left|\alpha_{1}\right\rangle=\frac{\left|\hat{v}_{3}\right\rangle+\left|\hat{w}_{3}\right\rangle}{\sqrt{2+2 c^{2 j}}}, \quad\left|\alpha_{2}\right\rangle=\frac{\left|\hat{v}_{3}\right\rangle-\left|\hat{w}_{3}\right\rangle}{\sqrt{2-2 c^{2 j}}}
$$

are orthogonal. In the basis where the atomic quantization axis is $\hat{u}_{3}$, the state $\left|\alpha_{1}\right\rangle$ is a superposition of the Zeeman substates $|j\rangle,|j-2\rangle,|j-4\rangle, \ldots$ and the state $\left|\alpha_{2}\right\rangle$ consists of the states $|j-1\rangle,|j-3\rangle,|j-5\rangle, \ldots$.

\section{One-dimensional case}

When the field consists of two counterpropagating plane waves, the field is always polarized in the $x y$ plane and the system is essentially one dimensional. The atomic quantization axis $\hat{u}_{3}=\hat{z}$ in which the linear couplings disappear is constant. In this frame, the two orthonormal dark states (4.1) are given in terms of the dark Bloch states

$$
\left|\hat{v}_{3}\right\rangle=e^{-i \xi J_{z}} e^{-i \theta J_{y}}|j,+j\rangle, \quad\left|\hat{w}_{3}\right\rangle=e^{-i \xi J_{z}} e^{+i \theta J_{y}}|j,+j\rangle .
$$

Since the space $|j\rangle,|j-2\rangle,|j-4\rangle, \ldots$ that contains $\left|\alpha_{1}\right\rangle$ and excludes $\left|\alpha_{2}\right\rangle$ now is fixed, $A_{12}=0$ and there is no coupling between the two dark states. The operator $\boldsymbol{U}$ is diagonal on the basis of the fixed internal states $|1\rangle$ and $|2\rangle$ with the potentials $U_{1}$ and $U_{2}$ as diagonal elements.

The vector potentials $A_{1}$ and $A_{2}$ are found by using the relations

$$
\begin{aligned}
& i\left\langle\hat{v}_{3}|\nabla| \hat{v}_{3}\right\rangle=\left\langle\hat{z}\left|e^{i \theta J_{y} \nabla} \nabla\left(\xi \boldsymbol{J}_{z}+\theta \boldsymbol{J}_{y}\right) e^{-i \theta J_{y}}\right| \hat{z}\right\rangle=j c \nabla \xi, \\
& i\left\langle\hat{v}_{3}|\nabla| \hat{w}_{3}\right\rangle=\left\langle\hat{z}\left|e^{i \theta J_{y}} \nabla\left(\xi \boldsymbol{J}_{z}+\theta \boldsymbol{J}_{y}\right) e^{+i \theta J_{y}}\right| \hat{z}\right\rangle=j c^{2 j-1} \nabla(\xi \\
& -i c)
\end{aligned}
$$

in the definition (4.1). The result is

$$
A_{1}=-j \frac{c+c^{2 j-1}}{1+c^{2 j}} \nabla \xi, \quad A_{2}=-j \frac{c-c^{2 j-1}}{1-c^{2 j}} \nabla \xi
$$

The scalar potentials of the two dark states are

$$
\begin{aligned}
& U_{1}=\frac{1}{2 m} \frac{\left(\nabla\left\langle\hat{v}_{3}\right|\right) \nabla\left(\left|\hat{v}_{3}\right\rangle+\left|\hat{w}_{3}\right\rangle\right)}{1+c^{2 j}}-\frac{A_{1}^{2}}{2 m}-\frac{1}{8 m}\left(\frac{\nabla c^{2 j}}{1+c^{2 j}}\right)^{2}, \\
& U_{2}=\frac{1}{2 m} \frac{\left(\nabla\left\langle\hat{v}_{3}\right|\right) \nabla\left(\left|\hat{v}_{3}\right\rangle-\left|\hat{w}_{3}\right\rangle\right)}{1-c^{2 j}}-\frac{A_{2}^{2}}{2 m}-\frac{1}{8 m}\left(\frac{\nabla c^{2 j}}{1-c^{2 j}}\right)^{2} .
\end{aligned}
$$

The last term in the two expressions accounts for the change in the normalization constant. The first term in $U_{1}$ and $U_{2}$ is determined by

$$
\begin{gathered}
\left(\nabla\left\langle\hat{v}_{3}\right|\right)\left(\nabla\left|\hat{v}_{3}\right\rangle\right)=\langle\hat{z}| e^{i \theta J_{y}}\left[\left(\nabla \xi J_{z}\right)^{2}+\left(\nabla \theta J_{y}\right)^{2} e^{-i \theta J_{y} \mid}|\hat{z}\rangle,\right. \\
\left(\nabla\left\langle\hat{v}_{3}\right|\right)\left(\nabla\left|\hat{w}_{3}\right\rangle\right)=\left\langle\hat{x}\left|e^{i \theta J_{y}}\left[\left(\nabla \xi J_{z}\right)^{2}-\left(\nabla \theta J_{y}\right)^{2}\right] e^{+i \theta J_{y}}\right| \hat{z}\right\rangle .
\end{gathered}
$$

The expectation values of the squares are

$$
\begin{gathered}
\left\langle\hat{z}\left|e^{i \theta J_{y}} J_{z}^{2} e^{i \theta J_{y}}\right| \hat{z}\right\rangle=\left(j^{2}-\frac{1}{2} j s^{2}\right) c^{2 j-2}, \\
\left\langle\hat{z}\left|e^{i \theta J_{y}} J_{y}^{2} e^{i \theta J_{y}}\right| \hat{z}\right\rangle=\left(\frac{1}{2} j-j^{2} s^{2}\right) c^{2 j-2},
\end{gathered}
$$

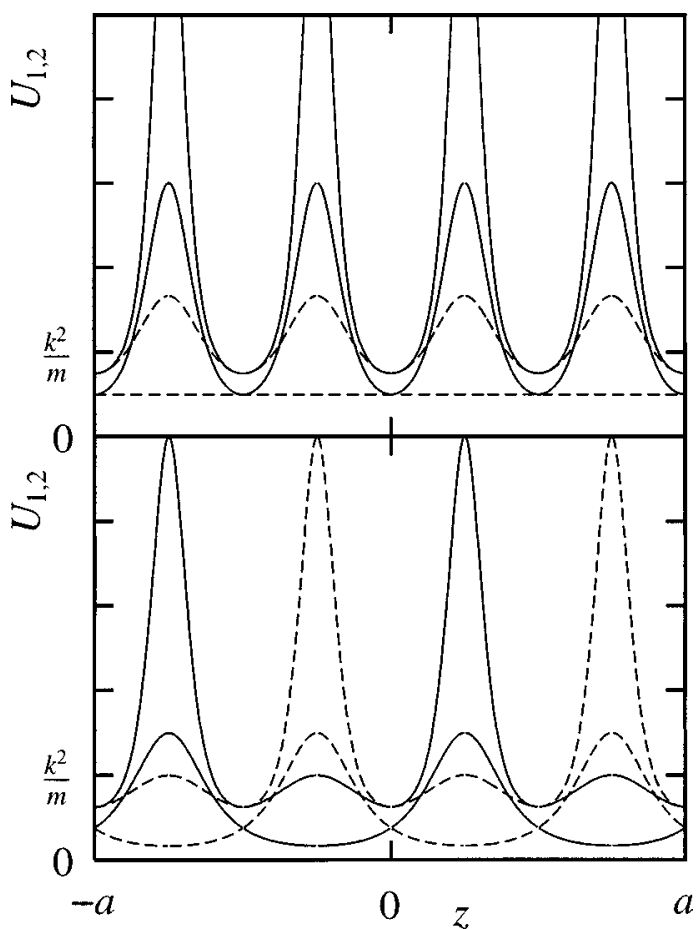

FIG. 4. The geometric potentials $U_{1}$ (solid) and $U_{2}$ (dashed) in the field with a fixed orientation and a uniformly varying ellipticity. The potential minima increase linearly with $j$. Top picture: integer values $j=1,2,3$. For $j=1, U_{1}=0$; for $j=1,2, U_{2}$ equals $k^{2} / 2 m$. Bottom picture: half-integer values $j=3 / 2,5 / 2$.

$$
\begin{gathered}
\left\langle\hat{z}\left|e^{i \theta J_{y}} \boldsymbol{J}_{z}^{2} e^{-i \theta J_{y}}\right| \hat{z}\right\rangle=j^{2} c^{2}+\frac{1}{2} j s^{2}, \\
\left\langle\hat{z}\left|e^{i \theta J_{y}} \boldsymbol{J}_{y}^{2} e^{-i \theta J_{y}}\right| \hat{z}\right\rangle=\frac{1}{2} j,
\end{gathered}
$$

Next to the $\operatorname{cosine} c=\cos \theta=\min (|\tan \varepsilon|,|\cot \varepsilon|)$, we abbreviated the sine $s=\sin \theta$. The two potentials $U_{1}$ and $U_{2}$ are evaluated in terms of the field parameters $\xi$ and $c$ by using Eq. (4.4) in Eq. (4.3) and substituting the result in Eq. (4.2). In turn, the field parameters are given by the expressions (3.8), (3.6), and (3.5) for an arbitrary field.

Now we turn to special fields that we choose as an example in Sec. III F. For a field with a fixed orientation like Eq. (3.16), $\xi=0$. Then

$$
\begin{aligned}
& U_{1}=\frac{j}{4 m}\left(\frac{1-c^{2 j-2}}{1+c^{2 j}}+\frac{2 j s^{2} c^{2 j-2}}{\left(1+c^{2 j}\right)^{2}}\right)(\nabla \theta)^{2}, \\
& U_{2}=\frac{j}{4 m}\left(\frac{1+c^{2 j-2}}{1-c^{2 j}}-\frac{2 j s^{2} c^{2 j-2}}{\left(1-c^{2 j}\right)^{2}}\right)(\nabla \theta)^{2} .
\end{aligned}
$$

We can use the explicit expressions (3.17) for the ellipticity. From the results we found that also these potentials have peaks at the intensity minima. The strengths of the peaks increase linearly with $j$. When $j>1$, extra peaks appear near the points where the field is circularly polarized. The potentials $U_{1}$ and $U_{2}$ are plotted in Fig. 4 for different $j$ values at $\alpha=\pi / 4$ when the two linear polarizations of the light beams are orthogonal. Then the field intensity is homogeneous and $\varepsilon(z)=k z$ so that the gradient $\nabla \varepsilon=k$ is constant. For integer vales of $J$, the potentials have a periodicity of $a / 2$, a quarter 
of an optical wavelength. For half-integer $j$ values, the two potentials $U_{1}$ and $U_{2}$ have the periodicity $a$, and are identical apart from a spatial shift of $a / 2$.

According to Eq. (2.8), the geometric potentials are proportional to the sum of the absolute squares of the nonadiabatic coupling constants $A_{i j}$. Hence optical pumping is maximal at the potential maxima. In the case that the two geometric potentials of the two dark states are different, the Sisyphus effect may be at work. When the atom is pumped from one dark state to the other dark state, most of the time the kinetic energy decreases until the steady state is reached. With each optical pumping cycle this net loss is of the order of $j$ times the recoil energy.

In the second example (3.18), the field is purely linearly polarized in each point. Then $c=\varepsilon=0$. Such a field is of the form (3.18), so that the polarization angle $\xi(z)$ is given in Eq. (3.19). The potentials (4.2) now reduce to

$$
U_{1}=U_{2}=\frac{j}{4 m}(\nabla \xi)^{2} \quad \text { if } \quad j>1
$$

For an arbitrary field in one dimension, when $j=2, j^{\prime}=1$, the potential $U_{2}$ equals Eq. (3.11), so that is explicitly given by Eq. (3.12) in terms of the field parameters. In the case $j=1, j^{\prime}=0$, the potential $U_{1}$ equals Eq. (3.11) and $U_{2}$ vanishes because the state $\left|\alpha_{2}\right\rangle$ is constant. This implies that the coupling to the other internal states is absent. A full quantum state with an arbitrary wave function $\psi(z)$ in a dark state with a vanishing geometric potential is black. There is no velocity selection so that cooling will not occur. When the geometric potential of a dark state is constant but nonzero, there are no forces, but there still is velocity selection in the nonadiabatic coupling to the excited state. In situations where finite families of states arise [10], both the light-shift potentials and the geometric potentials are flat. Like the driven $1 \rightarrow 1$ transition, the systems $1 \rightarrow 0$ and 2 $\rightarrow 1$ in one dimension contain a black state that is an eigenstate of the geometric potential.

\section{KRONIG-PENNEY MODEL}

In a dark state, the geometric potential $U$ depends on the polarization pattern and not on any other experimental parameter such as the detuning or field intensity. Although the expression (3.3) is given in terms of the electric field, the overall strength of the light field intensity $I$ drops out. Apart from the recoil factor $k^{2} / 2 m$ that gives $U$ the physical dimension of energy, it contains only geometrical variables of the electric field. This justifies the name geometric potential for $U$. Because the kinetic energy of a localized atom always exceeds the recoil energy, the effects of the geometric potential on the atomic motion are expected to be small. However, the geometric potential varies with $z$ proportional to the inverse square of the local field intensity $E^{4}$. Hence still large values can be expected at the intensity minima.

In order to demonstrate this, we consider the dark state in the $\Lambda$ 's of the $1 \rightarrow 1$, the $1 \rightarrow 0$, and the $2 \rightarrow 1$ transitions in an arbitrary field in one dimension. Then, and for the case of $j \rightarrow j-1$ in purely linearly polarized light, the vector potential is proportional to the function

$$
\begin{aligned}
\frac{\sin 2 \alpha}{1-\cos 2 \alpha \cos 2 k z} & =\sum_{n} \frac{\gamma}{\gamma^{2}+(k z+\pi n)^{2}} \\
& =\sum_{n} e^{2 i n k z} e^{-2|n| \gamma}
\end{aligned}
$$

with the parameter $\tanh 2 \gamma=\sin 2 \alpha$. The summations of $n$ extend over all integers. The scalar potential is proportional to the square of this function. Hence the vector potential is represented as a series of displaced Lorentz-type profiles in space. For small $\alpha<\gamma \ll 1$, this function becomes a series of narrow peaks and the Fourier components $\exp (-2|n| \gamma)$ go to the constant value 1 . The peaks are located at the intensity minima of the field. The rapid variation of the polarization causes large values of the geometric potential at these points.

\section{Dispersion relation}

For small angles the light-shift potential in the bright state (3.10) and the geometric potentials (3.12) are approximated by

$$
V_{2}(z)=\frac{\kappa^{2} I}{2 \delta} \sin ^{2} k z, \quad U(z)=\frac{k^{2}}{2 m} S \sum_{n} \delta(k z+n \pi) .
$$

This potential defines the well-known Kronig-Penney model. It is the only known model for a periodic potential that can be solved analytically. This may be a mere toy model when applied to describe electronic waves in the solid state; the present case of cold atoms in a dark state constitutes a physical realization of this model. The strength of $U$ equals $S$ $=j \pi / 2 \alpha$ for the $j \rightarrow j-1$ transition in purely linear polarized light and $j>1$ and $S=\pi \beta^{2} / \alpha^{3}$ for the $1 \rightarrow 1,1 \rightarrow 0$, and 2 $\rightarrow 1$ systems. The geometric potential diverges when the field has nodes. This occurs when $\vec{E}_{+}=-\vec{E}_{-}$and $\alpha=0$. However, when $\alpha=0$, the field polarization is fixed and only changes sign at the nodes. Then there is no geometric potential at all. The paradox is resolved by noting that when $\alpha$ approaches zero, the light-shift potential of the bright state $V_{1}$ and of the dark state $V_{0}=0$ at the intensity minima become degenerate. Landau-Zener coupling can occur and the atomic internal state is no longer restricted to the dark state. However, as long as the Doppler shift $p k / m$ is small compared to the minimal energy splitting $\kappa^{2} E^{2}(0) / \delta$ $=2 \kappa^{2} I \alpha^{2} / \delta$, the adiabatic approximation still holds. Even for small values of $\alpha$, the Rabi frequency $\kappa^{2} I / \delta$ can still be made sufficiently large to fulfill this condition.

If we consider a plane atomic wave incident on a single $\delta$ peak of the periodic potential, the wave is partly transmitted and partly reflected. When the wave has the wave vector $q$ so that the energy is $E=q^{2} / 2 m$, the transmission and reflection coefficients $t$ and $r$ are determined by

$$
\frac{1}{t}=\frac{1}{1+r}=1+\frac{i k S}{2 q} .
$$

The reflection coefficient $r$ is close to unity if $q \ll k S$, which is the case in the present approximation $\alpha \ll 1$ for moderate values of $q$. Atoms are reflected at the points where the field almost has a node. Hence atoms can be confined between the nodal planes of the field. The potential $U$ has discrete 
maxima at the intensity minima. Periodic electric fields in more dimensions have nodal lines and points instead of nodal planes. Therefore, bound states do not seem to occur in optical lattices in more dimensions. Fields with curved nodal planes are needed to trap in three dimensions. Possible candidates of such fields are Gaussian standing waves and spherical waves. This could be of interest for the study of collective effects. In order to confine atoms in the dark state, a binding potential is needed to contain an atomic sample of high density. When the atoms interact, the gauge potential may be used for evaporative cooling. Also this cooling mechanism is compatible with VSCPT since the ground state is black.

In the limit of small angles, the peaks are very narrow and strong. Periodic potentials, however, also give rise to a spectrum with energy bands. The dispersion relation between energy $E=q^{2} / 2 m$ and the quasimomentum $p$ for the KronigPenney model [12] is

$$
\cos p a=\cos q a-\frac{1}{2} \pi S \text { sinc } q a .
$$

Real values for $p$ can only be found where $q a=N \pi+\varepsilon$ and $\varepsilon$ of order $\alpha$. Then the energy bands are given explicitly by

$$
E_{N}(p)=\frac{k^{2}}{2 m}\left[N^{2}+\frac{4 N}{\pi S}(-)^{N} \cos p a\right] .
$$

Here $p$ is the quasimomentum and $N=1,2, \ldots$ is the band number. The half-bandwidth is the rate at which localized Wannier states tunnel to the neighboring wells [12] and is inversely proportional to the potential strength $S$.

When the black state lies in a continuum of energy states, as is the case when $U$ is periodic, then VSCPT cooling is governed by Lévy statistics [13]. The trapping process is characterized by the very slow growth of population $\sqrt{\Gamma t}$ in the black state, where $\Gamma$ is an effective escape rate. This is caused by the fact that, after a spontaneous emission, the overlap of the atomic wave function with the black state is infinitesimal. In the presence of a binding potential, the localized bound states have a discrete spectrum. Then the overlap with the black state after a photon emission is finite and the trapped population is expected to increase exponentially to unity.

In the regime where $\alpha$ is small, the bound Wannier states are approximate eigenstates. We expect that trapping in the first Wannier state occurs exponentially fast. This is followed by a process of tunneling and localization by spontaneous emissions. This dynamics in the first energy band is modeled in [14].

\section{CONCLUSIONS}

When differences between light-shift potentials exceed the Doppler shift of a moving atom, the atomic state will remain confined in a single position-dependent adiabatic internal state. Radiative forces do not arise only from the spatially varying light shifts, but also from spatial gradients in the internal state. As shown in [2], this effect is described by a geometric scalar and vector potential in the adiabatic approximation. We show that this can generally be described by a vector potential operator. This vector operator has simi- lar physical effects to an external magnetic field on a charged particle. In particular, it gives rise to a Lorentz-type force and, for a quantum mechanical wave packet, to Berry's topological phase. For a periodic potential in one dimension, this Berry phase determines the quasimomentum of the Bloch states.

In the adiabatic approximation, the internal state follows the atomic position. Then the square term in the general vector operator gives rise to the scalar potential. This potential energy can be interpreted as the kinetic energy contained in the spatial variation of the internal state.

Quantum motion becomes important when spatial coherences are of the order of an optical wavelength. In a dark internal state, coherences are preserved longer since spontaneous emission rates are small. Moreover, the subrecoil cooling mechanism VSCPT creates large spatial coherences in the dark state. In a dark state, where the light-shift potential vanishes, the geometric potential will be the dominant term. This implies that optical lattices will form naturally in a dark state. For configurations with a dark state, an external magnetic field can create gray lattices based on the magnetooptical potential. Whereas in gray lattices there is still a small coupling to the excited state, in a dark lattice, the excited state is decoupled.

In the transition between two levels with $j=1$, a single dark state exists for arbitrary polarization. We evaluate the dark geometric potentials for an arbitrary field in three dimensions. In one dimension, the geometric potential has a universal shape, which is inversely proportional to the square of the field intensity pattern. It has peaks at the intensity minima. The black state, which is a dark eigenstate of the total Hamiltonian, is also an exact eigenstate of the adiabatic Hamiltonian.

For a transition $j \rightarrow j-1$, two dark internal states exist. We evaluate the two dark geometric potentials in one dimension for arbitrary $j$ values. Apart from the peaks at the intensity minima, extra peaks appear at the points where the field is purely circularly polarized. The peak heights are proportional to $j$. In general, there will be a small velocity selective coupling to the excited level in the dark state. Transitions between the geometric potentials of two dark states can lead to Sisyphus cooling, thereby putting the atoms in the lowestenergy states of the geometric potential.

Systems with $j^{\prime}=j$ have a single dark state for integer $j$. We have considered only the case $j=1$, but we expect a nontrivial potential for large values of $j^{\prime}=j$, just as for the situation $j^{\prime}=j-1$ and $j^{\prime}=j=1$. Also a single dark state may lead to cooling. If the detuning $\delta$ is negative, all the light-shift potentials are positive. Atoms can only leave the dark state by moving nonadiabatically to the bright states [11]. When the Doppler shift $\mathrm{kp} / \mathrm{m}$ is small compared to the bright potential $V_{1}$, this effect is a first-order correction to the adiabatic approximation. Optical pumping from the bright state with a large positive potential energy $V_{1}+U_{1}$ to the dark state with a small potential energy $U_{0}$ will result in a net loss of energy. This process can be seen as a kind of Sisyphus cooling via a Landau-Zener transition from the dark to the bright state.

The geometric potential does not destroy the VSCPT cooling mechanism. In fact, we show that when a full black state exists, it lies in the lowest-energy band of the periodic 
geometric potential. Therefore, Sisyphus cooling can be effective in the subrecoil domain, which would increase the trapping in the black state. In one dimension, isolated black states are found in the $1 \rightarrow 1,3 / 2 \rightarrow 1 / 2$, and $2 \rightarrow 1$ systems. The periodic geometric potentials of these systems are equal. In configurations with field nodes, it diverges. We show that the adiabatic approximation can still be valid with intensity minima that are small compared to the intensity maxima. In this situation, the atom-field configuration constitutes a real- ization of the Kronig-Penney model with $\delta$-peaked potential barriers. The band structure is expressed in terms of the field parameters.

\section{ACKNOWLEDGMENTS}

This work is part of the research program of the Foundation for Fundamental Ressearch on Matter (FOM) and was made possible by financial support from the Netherlands Organization for Scientific Research (NWO)
[1] A. Aspect, E. Arimondo, R. Kaiser, N. Vansteenkiste, and C. Cohen-Tannoudji, Phys. Rev. Lett. 61, 826 (1988).

[2] R. Dum and M. Ol'shanii, Phys. Rev. Lett. 76, 1788 (1996).

[3] V. Taichenachev, A. M. Tumaikin, V. I. Yudin, and G. Nienhuis, Zh. Eksp. Teor. Fiz. 108, 415 (1995) [JETP 81, 224 (1995)].

[4] A. V. Bezverbnyi, G. Nienhuis, and A. M. Tumaikin, Opt. Commun. 148, 151 (1998).

[5] E. Arimondo and G. Orriols, Lett. Nuovo Cimento 17, 33 (1976).

[6] A. M. Tumaikin and V. I. Yudin, Zh. Eksp. Teor. Fiz. 98, 81 (1990) [Sov. Phys. JETP 71, 43 (1990)].

[7] G. Grynberg and J.-Y. Courtois, Europhys. Lett. 27, 41 (1994).

[8] M. A. Ol'shanii and V. G. Minogin, in Light Induced Kinetic Effects on Atoms, Ions and Molecules, edited by L. Moi, S.
Gozzini, C. Gabbanini, E. Arimondo, and F. Strumia (Ets Editrice, Pisa, 1990).

[9] A. V. Taichenachev, A. M. Tumaikin, M. A. Ol'shanii, and V. I. Yudin, Pis'ma Zh. Eksp. Teor. Fiz. 53, 366 (1991); [JETP Lett. 53, 351 (1991)].

[10] P. M. Visser and G. Nienhuis, Quantum Semiclassic. Opt. 9, 621 (1997).

[11] A. P. Kazantsev, G. I. Surdutovich, and V. P. Yakovlev, Mechanical Action of Light on Atoms (World Scientific, Singapore, 1990).

[12] N. W. Ashcroft and N. D. Mermin, Solid State Physics (HoltSaunders, Philadelphia, 1976).

[13] F. Bardou, J. P. Bouchard, O. Emile, A. Aspect, and C. CohenTannoudji, Phys. Rev. Lett. 72, 203 (1994).

[14] P. M. Visser and G. Nienhuis, Phys. Rev. A 56, 3950 (1997). 